\title{
Applicability of Remote Sensing Workflow in Kubernetes-Managed On-premise Cluster Environment ${ }^{+}$
}

\author{
Hemang Narendra Vithlani *, Dogotari Marcel, Bethany Melville, Moritz Prüm, \\ Olee Hoi Ying Lam, Rolf Becker and Frank Zimmer
}

Faculty of Communication and Environment, Rhine-Waal University of Applied Sciences, Hochschule RheinWaal, 47475 Kamp-Lintfort, Germany; DOM@hsrw.eu (D.M.); BME@hsrw.eu (B.M.); MPR@hsrw.eu (M.P.);

LH@hsrw.eu (O.H.Y.L.); RB@hsrw.eu (R.B.); FZ@hsrw.eu (F.Z.)

* Correspondence: HNV@hsrw.eu

+ Presented at TERRAenVISION 2019, Barcelona, Spain, 2-7 September 2019.

Published: 24 December 2019

\begin{abstract}
The acquisition, storage, and processing of huge amounts of data and their fast analysis to generate information is not a new approach, but it becomes challenging through smart decisionmaking on the choice of hardware and software improvements. In the specific cases of environment protection, nature conservation, and precision farming, where fast and accurate reactions are required, drone technologies with imaging sensors are of interest in many research groups. However, post-processing of the images acquired by drone-based sensors such as the generation of orthomosaics from aerial images and superimposing the orthomosaics on a global map to identify the exact locations of the interested area is computationally intensive and sometimes takes hours or even days to achieve desired results. Initial tests have shown that photogrammetry software takes less time to generate an orthomosaic by running them on a workstation with higher CPU, RAM and GPU configurations. Tasks like setting up the application environment with dependencies, making this setup portable and manage installed services can be challenging, especially for small-andmedium-sized enterprises that have limited resources in exploring different architectures. To enhance the competitiveness of the small and medium-sized enterprises and research institutions, the accessibility of the proposed solution includes the integration of open-source tools and frameworks such as Kubernetes (version v1.13.4, available online: https://kubernetes.io/) and OpenDroneMap (version 0.3, available online: https://github.com/OpenDroneMap/ODM) enabling a reference architecture that is as vendor-neutral as possible. Current work is based on an onpremise cluster computing approach for fast and efficient photogrammetry process using open source software such as OpenDroneMap combined with light-weight containerization techniques such as Docker (version 17.12.1, available online: https://www.docker.io/), orchestrated by Kubernetes. The services provided by OpenDroneMap enable microservice-based architecture. These container-based services can be administered easily by a container orchestrator like Kubernetes. After setting up the servers with core OpenDroneMap services on our container-based cluster with Kubernetes as the orchestrator engine, the plan is to use the advantages of Kubernetes' powerful management capabilities to help maximize resource efficiency as the basis for creating Service Level Agreements to provide a cloud service.
\end{abstract}

Keywords: Remote Sensing; Cluster Computing; Orthomosaic; OpenDroneMap; Kubernetes 
Acknowledgments: This work is part of the SPECTORS project with project number 143081, which is funded by the cooperation program INTERREG Deutschland-Nederland. The authors would like to acknowledge the support of teuto.net Netzdienste $\mathrm{GmbH}$ in Kubernetes consultation, and the support of KnowH2O and Wageningen University \& Research in defining a use-case for the system.

(C) 2019 by the authors. Licensee MDPI, Basel, Switzerland. This article is an open access article distributed under the terms and conditions of the Creative Commons Attribution (CC BY) license (http://creativecommons.org/licenses/by/4.0/). 